\title{
Generation iUltrasound: Bedside diagnostic imaging with Hand-Held Cardiac Ultrasound
}

\author{
Amer M. Johri \\ Cardiovascular Imaging Network Queen's, Queen's University Kingston Ontario, Canada
}

"I would have gladly attended the arterial concert." (Curveilhier to Laennec, 13 April 1824 [1])

In the early nineteenth century, an enquiring physician rolled up a notebook and placed it upon the chest of a young woman in order to hear her heart and understand its rhythmic beating more clearly. Thus began the teaching of a new approach to 'les maladies cardiologiques' [1]. Two-hundred years later this tube remains the cornerstone of the cardiac physical exam; an object evocative of our profession (Figs. 1, 2).

As clinical educators, are we ready for a change in diagnostic technology? Whether we are or not, the availability of small, portable Hand-Held Cardiac Ultrasound (HHCU) devices portends a tidal change in diagnostic ability with important implications to the physical exam and point-of-care diagnosis (Fig. 3).

We are now tasked with establishing the capabilities and limitations of these new medical devices in the hands of various users. Some consensus is emerging: studies have shown that these devices are a valuable tool for augmenting the diagnostic value of the standard physical exam and even novel learners can be taught to identify specific cardiac pathologies using HHCU [2-4]. However it is now clearly recognized that bedside examination with HHCU is not to be considered a substitute for a comprehensive or limited echocardiogram, and with the current technology these machines are only suited to performing focused cardiac ultrasound examinations, even when used by expert sonographers [5]. Within the practice of focused cardiac ultrasound, the image acquisition and interpretation skills of the user are the major factors determining the efficacy of these devices as a mechanism for improving the diagnostic power of a standard cardiac exam [6].

Further research into the development and implementation of HHCU training is warranted. The Cardiovascular Imaging Network at Queen's
(CINQ) has sought to gain experience in developing a critical framework for teaching HHCU skills in new learners. Our group is led by expert Level III trained echocardiographers with expertise in medical education. Our work has been presented at several conferences across Canada and the USA [2, 7] and we lead the Canadian National Working Group on HHCU Education. We recently advanced this work by conducting a preliminary study in 10 senior internal medical residents demonstrating that $\mathrm{HHCU}$ improved clinical management of patients in the Emergency Department and improved the confidence of these physicians in their assessment of patients referred for cardiology assessment [8]. Although this exciting preliminary work shows feasibility of a formal HHCU training program for residents, careful further study is required to investigate the optimal method of delivering this integrative skill.

Acquisition of HHCU skill is becoming recognized as an emerging essential competency, however, currently medical residency has objectives set by the Royal College which do not incorporate these specific skills. Thus, additional training of this complex integrative skill requires recognition of the current time and resource constraints placed upon learners and educators. To address these challenges, we developed rich electronic modules in coordination with our Information Technology experts at Queen's School of Medicine, and worked with our Simulation Laboratory to acquire and develop a state-of-the-art cardiac ultrasound simulation program. In addition to the lecture based curriculum developed by expert educators in our laboratory, we developed resources available for delivering $\mathrm{HHCU}$ programming in post-graduate training. We now intend to look at various combinations of these resources to determine the optimal method of delivering HHCU skill to internal medicine trainees during their busy residency. 


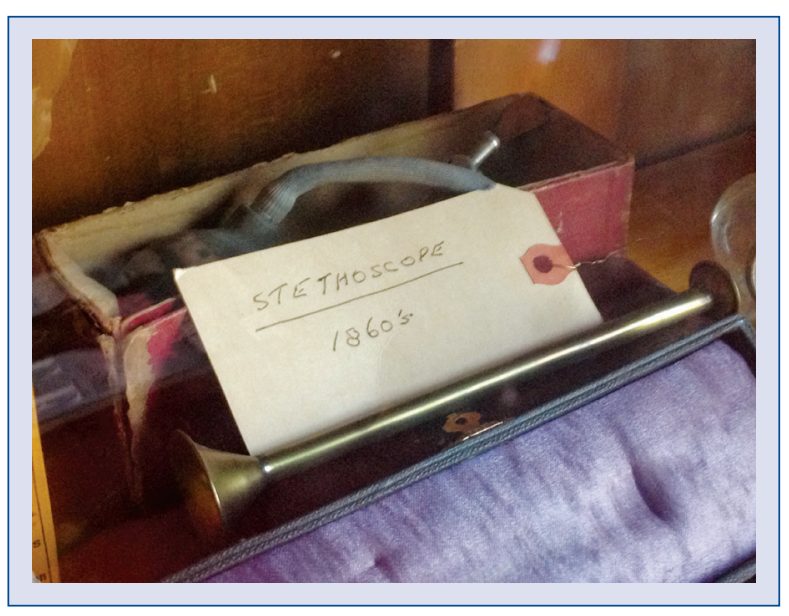

Figure 1. 1860's stethoscope [10].

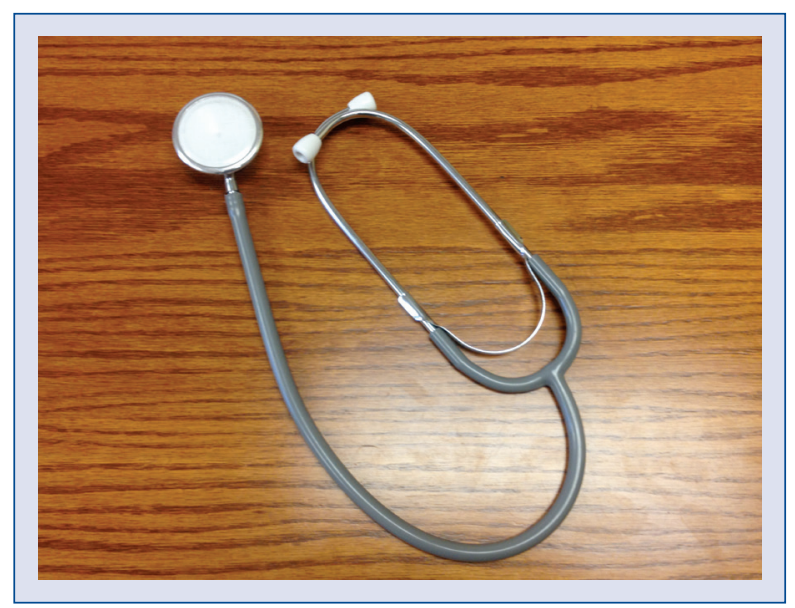

Figure 2. Modern stethoscope [10].

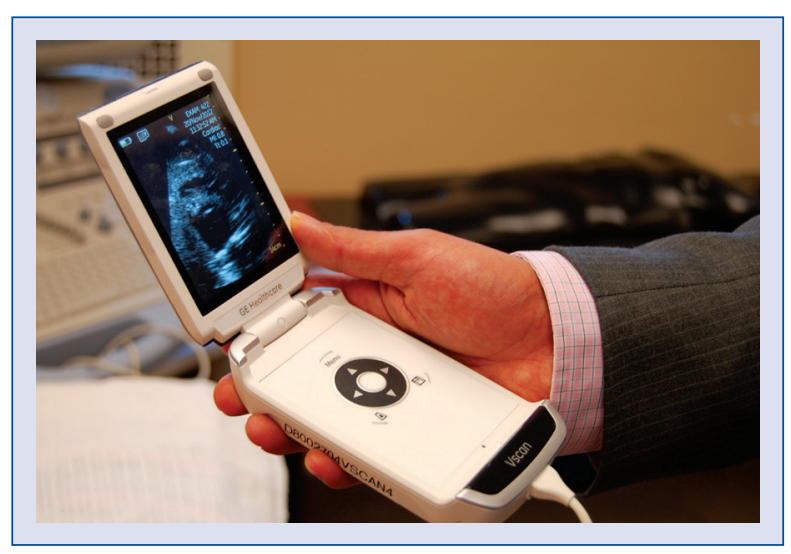

Figure 3. GE Vscan - Hand-Held Cardiac Ultrasound device [10].

To ensure that we develop a critical framework of learning in not only HHCU but focussed ultrasound of other systems, collaboration amongst the various clinical fields including cardiology, anesthesia, emergency and internal medicine is required. With respect to HHCU, imaging societies suggest training to occur under the auspices of a designated expert, such as an echocardiographer with Level III training to ensure on-going quality control and maintenance of skills [9]. HHCU devices are now used 'ad hoc' by some physicians of various backgrounds, despite little being known about the efficacy, safety, or training benchmarks required to implement such technology by non-echocardiographers. Nor is there a consensus from any national or international body regarding the timing or method of training for HHCU in post-graduate medical education. There is a significant lack of quality control in this area, as well as programs to ensure that learned skills are maintained over time. As clinical educators, we cannot alter the tide, but must gain the forefront to ensure our trainees learn within a well-developed framework; optimizing use of HHCU while remaining within scope.

Disclosures: Partial funding for VScan devices used by CINQ provided by GE Healthcare Equipment Grant.

Conflict of interest: Dr Amer Johri (Author) has previously collaborated with Dr Baranchuk (Deputy Editor) in various research endeavours as colleagues within the Division of Cardiology at Queen's University.

\section{References}

1. Duffin J. To see with a better eye: A Life of R.T.H. Laennec. Princeton University Press, Princeton, New Jersey 1998.

2. Nickel C, Sanfilippo A, Johri AM. Incorporation of the Hand Held Ultrasound into the Clinician's Toolkit: Can novel learners be trained to identify cardiac pathology with hand held ultrasound? Canadian J Cardiol, 2012; 28: 302.

3. Panoulas VF, Diageler A-L, Malaweera ASN, Lota AS, Baskaran D, Nihoyannopoulos P. Pocket-sized Hand-Held Cardiac Ultrasound as an adjunct to clinical examination in the hands of medical students and junior doctors. Eur Heart J Cardiovas Imaging, 2013; 14: 323-330.

4. Cawthorn TR, Nickel, C, O'Reilly M et al. Development and evaluation of methodologies for teaching focused cardiac ultrasound skills to medical students. J Am Soc Echocardiogr, 2014; Jan 13 [Epub ahead of print].

5. Sicari R, Galderisi M, Voigt JU et al. The use of pocket-size imaging devices: A position statement of the European Association of Echocardiography. Eur J Echocardiogr, 2011; 12: 85-87.

6. Alexander JH, Peterson ED, Chen AY, Harding TM, Adams DB, Kisslo JA, Jr. Feasibility of point-of-care echocardiography by internal medicine house staff. Am Heart, 2004; 147: 476-481.

7. Cawthorn TR, Sanfilippo A, Johri AM. Development of a curriculum for training medical students in the use and interpretation of hand-held echocardiography. Canadian J Cardiol, 2012; 28: S302-S303.

8. McIntyre WF, Gilmour JA, Smith CA, Baranchuk A, Johri AM. Can hand-held cardiac ultrasound improve patient management in the emergency department? Application of Point-of-Care Technology by Internal Medicine Residents. Abstract Accepted to $24^{\text {th }}$ Annual American Society of Echocardiography Scientific Sessions, 2013.

9. Spencer KT, Kimura BJ, Korcarz CE et al. Focused cardiac ultrasound: Recommendations from the American Society of Echocardiography. J Am Soc Echocardiogr, 2013; 26: 567-581.

10. Cardiovascular Imaging Network Queen's Image Library, 2013. 